\title{
COMPREHENSIVE GROUP THERAPY OF OBESITY AND ITS IMPACT ON SELECTED ANTHROPOMETRIC AND POSTURAL PARAMETERS
}

\author{
Stanislav Horák ${ }^{1,2}$, Eliška Sovová ${ }^{3}$, Dalibor Pastucha ${ }^{4,5}$, Petr Konečný2, 6,7, Lenka Radová8, Naděžda Calabová1, ${ }^{1,}$ \\ Jana Janoutová', Vladimír Janout ${ }^{9}$ \\ ${ }^{1}$ Department of Rehabilitation, University Hospital Olomouc, Olomouc, Czech Republic \\ ${ }^{2}$ Department of Physiotherapy, Faculty of Health Sciences, Palacký University Olomouc, Olomouc, Czech Republic \\ ${ }^{3}$ Department of Exercise Medicine and Cardiovascular Rehabilitation, University Hospital Olomouc and Faculty of Medicine and Dentistry, \\ Palacký University Olomouc, Olomouc, Czech Republic \\ ${ }^{4}$ Department of Rehabilitation, Faculty of Medicine, University of Ostrava, Ostrava, Czech Republic \\ ${ }^{5}$ ReFit Clinic, Olomouc, Czech Republic \\ ${ }^{6}$ Department of Rehabilitation, Prostëjov Hospital, Prostějov, Czech Republic \\ ${ }^{7}$ Department of Physiotherapy and Rehabilitation, Faculty of Medicine, Masaryk University, Brno, Czech Republic \\ ${ }^{8}$ Central European Institute of Technology, Masaryk University, Brno, Czech Republic \\ ${ }^{9} \mathrm{Centre}$ for Research and Science, Faculty of Health Sciences, Palacký University Olomouc, Olomouc, Czech Republic
}

\section{SUMMARY}

Objective: Obesity is a multifactorial disease. This non-infectious epidemic has reached pandemic proportions in the 21 century. Posture is a dynamic process referring to an active maintenance of body movement segments against the action of external forces. The aim of the study was to investigate the effect of comprehensive group therapy for obese persons on selected anthropometric and postural parameters.

Methods: The study comprised 53 females with a mean age of 44.5 years (range 29-65 years, standard deviation 9.42 years, median 44 years), who completed a controlled weight loss programme. At the beginning and at the end of the programme, anthropometric parameters (Body Mass Index (BMI), weight and waist circumference) were measured and the posturography tests Limits of Stability (LOS) and Motor Control Test (MCT) were performed using the NeuroCom's SMART EquiTest system. The data were statistically analyzed using R software at a level of significance of 0.05 .

Results: There were positive changes after the controlled weight loss programme in anthropometric parameters (BMl reduction, with $p<0.001$; waist circumference reduction, with $p<0.001$; and weight loss, with $p<0.001$ ), postural stability with statistically significant $(p<0.05)$ improvements in both postural activity (LOS test parameters) and reactions (MCT parameters).

Conclusion: The study showed a statistically significant effect of comprehensive group therapy for obesity in terms of reductions in waist circumference, body weight and BMI, and thus the overall reduction of both cardiovascular and metabolic risks, as well as improved postural skills (activity and reactions).

Key words: body mass index, obesity, waist circumference, posture, posturography, controlled weight loss programme

Address for correspondence: S. Horák, Department of Rehabilitation, University Hospital Olomouc, I. P. Pavlova 6, 77520 Olomouc, Czech Republic. E-mail: stanislav.horak@fnol.cz

https://doi.org/10.21101/cejph.a4780

\section{INTRODUCTION}

Obesity is considered one of the most serious lifestyle diseases, reaching pandemic proportions in the 21 st century. It is usually determined by multiple factors. The condition affects an individual's life both qualitatively and quantitatively $(1,2)$.

Body mass index (BMI) is a simple measure easy to calculate using a person's height and weight. This simplicity, however, is linked to inaccuracy and thus disadvantages (3). There are numerous factors that negatively influence the potential use of BMI to assess an individual's constitution. A single measure and set of defined values cannot be used to evaluate the entire population even though certain ranges have been defined. It is necessary to consider the assessed person's age, gender, overall physical constitution, and others $(4,5)$. Waist circumference is a separate measure and risk factor. It is one of the major criteria for assessing the presence of metabolic syndrome (MS). It indicates the amount of abdominal fat which, unlike subcutaneous adipose tissue, is metabolically active and thus poses a significant risk. The International Diabetes Federation has stressed the importance of central obesity as the key factor behind MS. The values are interpreted with respect to gender. Normal waist circumference is less than $94 \mathrm{~cm}$ for males and less than $80 \mathrm{~cm}$ for females. Values above $102 \mathrm{~cm}$ in males and $94 \mathrm{~cm}$ in females are associated with significant cardiometabolic risk $(1,6)$. 
Posture is a dynamic process of maintaining the position of the body and its parts before and after movement (7). Posture refers to active maintenance of the body's movement segments against the action of external forces (8). It accompanies the beginning and end of any intentional movement of a person. At the same time, it is a component of and an essential prerequisite for movement (9). Posture is assessed with computerized dynamic posturography (CDP). This technique is used to quantify postural stability and reactions. It is based on measuring reaction forces and moments using a force plate during static and dynamic situations. Specifically, it measures resolution of forces in three planes which are perpendicular to each other (8). Posturography may analyze both static and dynamic components of postural stability. Thus, it is a kinetic method, one that evaluates motion with regard to forces that cause it. The CDP SMART EquiTest system is used to assess standing balance under various conditions. The CDP SMART Balance Master is used to quantify walking balance $(10,11)$.

Only few articles have been published on comprehensive group therapy for obesity and the relationship with postural skills of an individual. Most authors have dealt with either obesity treatment or a person's postural skills. The effect of obesity on postural stability was investigated, for example, by Teasdale et al. in a study on postural stability before and after weight loss. The results showed that weight loss improved balance control and that the improvement was directly related to the amount of weight loss. Following their weight loss, individuals maintained stable positions for a longer time and the overall response of their postural control system improved (12). King et al. studied the effect of selected parameters, BMI, body fat percentage, moment of inertia and leg strength on postural control. They found that in obese people, reduced fitness led to a decrease in the ability to regulate increased demands on postural control. They suggested that the negative effect is more pronounced if an individual is close to their stability limits as compared to standing still. The authors pointed to the fact that obesity influences a person's balance and postural control not only by changes in physical parameters such as weight, BMI or body composition but also by diminished physical strength of the individual (13).

There is practically no research on the effect of comprehensive group therapy for obesity with respect to anthropometric parameters including cardiovascular risk as well as to postural parameters.

The aim of the study was to investigate the effect of comprehensive group therapy for obese persons on selected anthropometric and postural parameters, in particular its impact on reduction of waist circumference as a key marker for metabolic syndrome and cardiovascular risk as well as on improving an individual's postural skills and thus its effect on the entire range of complications resulting from impaired postural stability and reactions.

\section{MATERIALS AND METHODS}

The study included 53 females (a mean age of 44.5 years) who completed a controlled weight loss programme at the Department of Exercise Medicine and Cardiovascular Rehabilitation, University Hospital Olomouc, from 2011-2014. The programme comprised a baseline comprehensive medical examination involving laboratory tests for metabolic and hormone disorders, as well as spirometry and cardiac stress tests to assess physical fitness and to determine the optimal heart rate for physical activity. Additionally, the Bodystat device was used to analyze body composition, that is, to measure body fat and basal metabolic rates.

Programme contains:

Week 1: Initial session

Week 2: Group intervention - motivation for lifestyle change

Week 3: Nutritional counselling - education on adequate dietary habits

Week 4: Physical activity - its role in weight loss

Weeks 5 to 7: Individual programme with optional consultations and individual exercise

Week 8: Follow-up examination - evaluating success of the weight loss programme, Bodystat

Week 26: Follow-up examination - evaluating success of the weight loss programme, Bodystat

For each participant (in our study), two measurements were carried out, at the beginning of the programme and eight weeks later. Basic anthropometric parameters were obtained using a measure, digital weighing scales and a tape measure. Postural parameters were measured with the following NeuroCom's SMART EquiTest CDP products:

- Motor Control Test (MCT) assesses the motor system's ability to restore postural stability after an unexpected external stimulus (small, medium and large magnitude of forward and backward translations of the force plate) (14). It determines the effectiveness of automatic postural reactions depending on the direction and magnitude of translation. Three degrees of translation are tested by three measurements each. Small translation corresponds to a threshold stimulus; medium and large translations to a suprathreshold stimulus.

The parameters tested by MCT are weight symmetry - mean distribution of body weight during translations (\%), latency - effectiveness of reaction to force plate translation ( $\mathrm{ms}$ ), and amplitude scaling - quantification of active force response to force plate translation for each extremity $(10,14)$.

In the present study, the latency parameter was used as it most accurately reflects an individual's postural reactions.

- Limits of Stability (LOS) express the maximum distance to which an individual's center of gravity may be shifted without loss of balance and the use of external support. The LOS test is performed for a total of eight directions (forward, right, backward, left, and diagonal - forward-right, backward-right, backward-left, and forward-left). The initial position of the centre of pressure (COP) is always the centre.

The parameters tested by LOS are reaction time (RT) - response to a sound signal (s); movement velocity (MVL) - speed of COP upon reaching a pre-defined point $(\% / s)$; endpoint excursion (EPE) - distance of the COP movement at first attempt to reach the limits of stability (\%); directional control (DCL) - control over direction of the COP movement (\%), with a value of $100 \%$ indicating a straight line; and maximum excursion (MXE) maximum shift of COP in particular direction (\%), equal to the limit of stability $(10,14)$.

The data were statistically analyzed using R software at a level of significance of 0.05 . 


\section{RESULTS}

After eight weeks, there were statistically significant changes in selected anthropometric parameters (Table 1).

The therapy resulted in statistically significant reductions in waist circumference (mean value before therapy $108.6 \mathrm{~cm}$, mean value after therapy $104.94 \mathrm{~cm}, \mathrm{p}<0.001$ ), body weight (mean value before therapy $98.72 \mathrm{~kg}$, mean value after therapy 94.81 $\mathrm{kg}, \mathrm{p}<0.001$ ) and BMI (mean value before therapy 35.45 , mean value after therapy $34.04, \mathrm{p}<0.001)$.

The assessment of postural parameters showed a positive effect of the comprehensive therapy with statistically significant results in the vast majority of the parameters.

The LOS test demonstrated a statistically significant change in the RT parameter, with statistically significant improvements in all directions $(p<0.05)$. In the MXE parameter, significant improvements were noted for seven directions $(p<0.05)$, the only statistically non-significant change was in direction 8 , i.e. forward-left $(p<0.053)$. The EPE parameter was statistically significantly improved in all directions $(p<0.05)$. In the DCL parameter, significant improvements were observed in seven directions $(p<0.05)$, improvement in direction 7 , i.e. left, was not statistically significant $(\mathrm{p}<0.099)$. The MVL parameter was statistically significantly improved in all directions $(\mathrm{p}<0.05)$. The overall evaluation of the LOS test, i.e. after comparison and statistical analysis of results of the individual test components, the mean values of results showed improvements in all parameters tested. The greatest and smallest improvements were noted for MVL (30.6\%) and MXE (7.4\%), respectively. The changes in the LOS parameters are summarized in Fig. 1.

The assessment of the MCT latency parameter showed improvements of the mean values for both extremities and all degrees of translation. The greatest improvement was observed for the left lower extremity and small backward translation (9\%), the smallest improvements were demonstrated for the left lower extremity and large forward translation (4\%), and for the right lower extremity and large backward translation (4\%). The changes in the MCT latency parameter are summarized in Fig. 2.

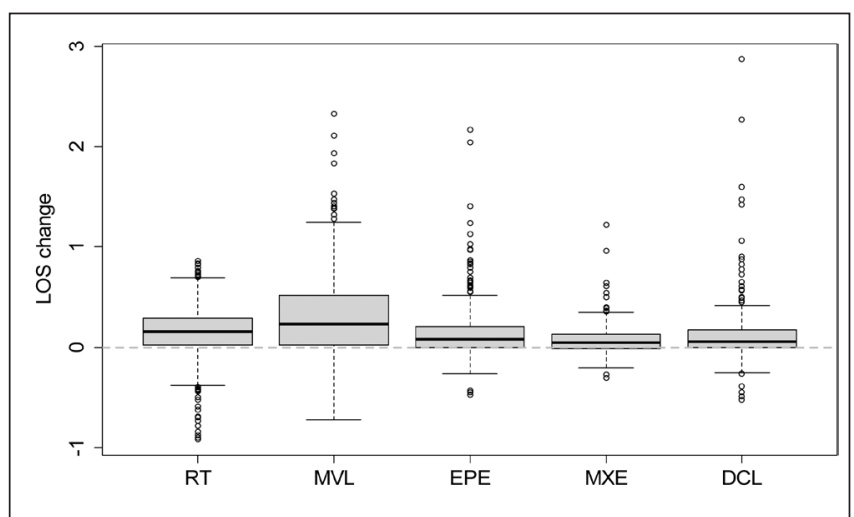

Fig. 1. Changes in individual LOS test parameters.

Values above 0 - improvement, below 0 - deterioration; bottom of the box $-25 \%$ quantile, top of the box $-75 \%$ quantile; band inside the box - median; circles - outliers; $\mathrm{RT}$ - reaction time, MVL - movement velocity, EPE - endpoint excursion, DCL directional control, MXE - maximum excursion

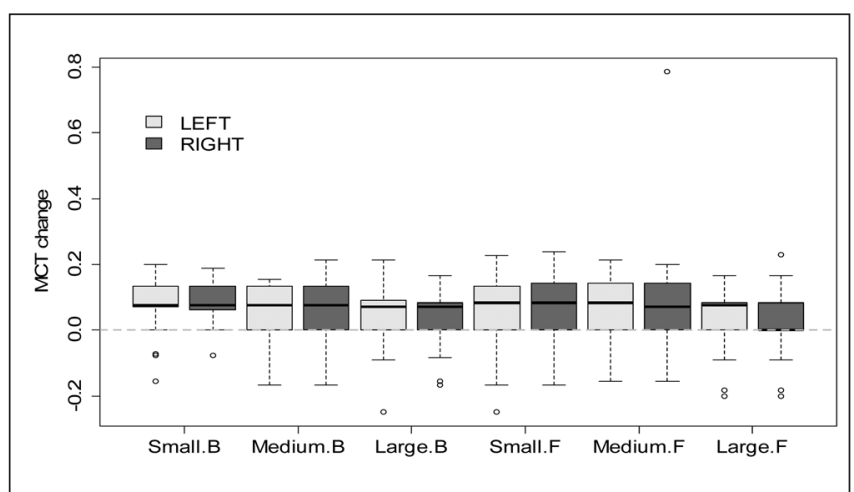

Fig. 2. Changes in MCT.

Values above 0 - improvement, below 0 - deterioration; bottom of the box $-25 \%$ quantile, top of the box $-75 \%$ quantile; band inside the box - median; circles - outliers: Small.B - small magnitude of backward translations of the force plate; Medium.B - medium magnitude of backward translations of the force plate; Large.B - large magnitude of backward translations of the force plate; Small.F - small magnitude of forward translations of the force plate; Medium.F - medium magnitude of forward translations of the force plate; Large.F - large magnitude of forward translations of the force plate

Table 1. Anthropometric characteristics of the sample $(N=53)$

\begin{tabular}{|l|c|c|c|c|c|c|c|c|}
\hline & Minimum & Maximum & Average & Variance & $\begin{array}{c}\text { Standard } \\
\text { Deviation }\end{array}$ & Median & $\mathbf{2 5 \%}$ quantile & $\mathbf{7 5 \%}$ quantile \\
\hline Age & 29 & 65 & 44.5 & 88.869 & 9.406 & 44 & 37 & 49 \\
\hline Height (m) & 1.5 & 1.8 & 1.7 & 0.004 & 0.061 & 1.7 & 1.6 & 1.7 \\
\hline $\begin{array}{l}\text { Weight before therapy } \\
\text { (kg) }\end{array}$ & 77.6 & 144.4 & 98.7 & 206.565 & 14.372 & 95 & 88.8 & 105.5 \\
\hline BMl before therapy & 30 & 50.8 & 35.5 & 23.258 & 4.823 & 34.6 & 31.5 & 37 \\
\hline Weight after therapy (kg) & 69.2 & 134.1 & 94.8 & 196.131 & 14.005 & 92.5 & 84.3 & 102 \\
\hline BMl after therapy & 27 & 48.9 & 34.0 & 21.838 & 4.673 & 33.2 & 30.8 & 35.9 \\
\hline Weight loss (kg) & -1.4 & 10.3 & 3.9 & 7.819 & 2.796 & 3.5 & 1.9 & 5.2 \\
\hline $\begin{array}{l}\text { Waist circumference } \\
\text { before therapy (cm) }\end{array}$ & 91 & 136 & 108.6 & 106.321 & 10.311 & 107 & 102 & 116 \\
\hline $\begin{array}{l}\text { Waist circumference } \\
\text { after therapy (cm) }\end{array}$ & 86 & 128 & 104.9 & 104.478 & 10.221 & 104 & 98 & 111 \\
\hline $\begin{array}{l}\text { Waist circumference } \\
\text { reduction (cm) }\end{array}$ & 0 & 11 & 3.7 & 7.882 & 2.808 & 3 & 2 & 5 \\
\hline
\end{tabular}




\section{DISCUSSION}

The study showed that completing a weight loss programme comprising not only dietary modifications but also implementation of a physical activity regimen resulted in improvements of both anthropometric and nearly all postural stability parameters. Unlike the present study including only females, a study by Ku et al. on the relationship between body weight and stability and the relationship between postural stability, BMI and gender in young adults showed an inverse association between postural stability and increasing BMI, and pointed to the fact that postural control is better in underweight persons, corresponding to the above findings, and that young females displayed a greater postural sway in the anterior-posterior and lateral directions than males (15). Both genders were also investigated by Cruz-Goméz et al. (16) who compared differences in the postural sway between males and females in various BMI categories during 4 conditions. Two measurements were made using a hard-surface force platform with subjects having their eyes opened and then closed. The other two measurements were made with subjects standing on a $5-\mathrm{cm}$ layer of foam rubber, once again with the eyes opened and then closed. Each measurement lasted 25.6 seconds. The participants were asked to stand upright, as still as possible, with their arms at their sides. The length and the area of oscillation and the displacement velocity of COP as a function of the anterior-posterior or lateral-lateral position. The study showed statistically significant increases in the length and area of sway in obese individuals standing on a hard surface. Closing the eyes produced a larger increase of sway in the obese subjects. When recordings were made on a soft surface, a larger area of sway was observed in the obese group, with these subjects being more dependent on vision to control balance (16).

The present study investigated and confirmed, among others, the effect of weight loss on improved postural stability. Similarly, a positive correlation between postural instability and BMI was demonstrated by Greve et al. reporting that a $20 \%$ increase in body mass reduced the ability to respond to external stimuli, leading to impaired postural stability. Patients with BMI greater than 30 maintained longer times unbalanced. Thus, obesity influenced the limits of postural stability. The authors also stated that obesity affected the selection of motor strategies employed to maintain postural balance (17). Another interesting study is the one by Handrigan et al. (18) stressing that the relationship between BMI and postural stability should be investigated also in athletes even though BMI values are generally considered as misleading in athletes. Included in the study were obese and heavy athletic subjects. The two groups had similar BMI but there were differences in muscular mass, strength and training habits. The aim was to determine the contribution of muscular strength to reducing postural sway. The results showed similar postural sway in both groups, suggesting that the amount of postural sway is not determined by lower limb muscular strength. This supports the hypothesis that body weight has a greater impact on postural sway than muscular strength. However, this conclusion is only true for individuals with BMI over 30 (18). Thus, the primary factor influencing postural sway in person with greater BMI is weight.

In the present study, the initial position for the assessment was quiet bipedal standing and the centre of gravity displacement was recorded using a computer with respect to the tested parameters and using the limits of stability. Comparison of postural sway during quiet standing was also investigated by Laughton et al. who found greater COP displacement in obese subjects as compared with lean ones (19). In their study of postural stability in morbidly obese persons, Singh et al. (20) used quiet upright standing supposed to last for one hour. All of the morbidly obese subjects failed to do the task completed by only six non-obese controls. Therefore, only the first twenty minutes of the assessment were analyzed. To objectify and quantify the ability to move the centre of gravity forward, the functional reach test was selected. That is, a simpler test using only one direction as compared to the present study. The arm was raised to reach 90 degrees and the maximum forward reach was measured without a change in the base of support. The resulting difference was statistically significant, with non-obese controls reaching a mean of 40.19 and morbidly obese subjects reaching only $32.25 \mathrm{~cm}$. Thus, consistently with the above studies, the ability to move the centre of gravity forward was reduced in morbidly obese individuals (20). By contrast, the use of posturography tests, as in the present study, was supported by Colné et al. (21), who reported that significant changes influencing balance in obese persons are linked to dynamic aspects of postural control. Additionally, the study showed that with the same COP position when standing, obese persons had less ability to sway forward or backward, as demonstrated by smaller COP shifts. The decrease in COP displacement is particularly striking for the backward sway as this is when COP is close to its position during quiet standing (21). Schieppati et al. (22) stated that testing the limits of stability is a better indicator for assessing postural stability than measurements of COP displacement only. It was found that in obese subjects, most parameters associated with COP dynamics are decreased. Upon increasing gait velocity, however, the same change in parameters occurred in both groups. Due to their excess weight, the overall performance is reduced in obese individuals, these are slower and have more difficulties controlling their vertical stability. To maintain stability, obese individuals choose to slow their movements (22). These limits of stability were also used in the present study, making the results more accurate. Different initial positioning was used in a study by Greve et al., showing a positive relationship between BMI and balancing on a single leg. They assessed 40 males whose postural stability was statistically significantly poorer if their BMI increased (17).

It should be mentioned that impaired postural stability is associated with a risk for falls, potentially leading to trauma and subsequent disability. The above finding that balance improves following weight loss were supported in a metaanalysis by Del Porto et al. (23). The authors also stated that the incidence of falls increases with body mass. Weight loss is able to reduce or even eliminate changes developed by obese persons as a result of adaptations to obesity. High levels of weight loss lead to significant improvements in both postural stability and relative strength. Weight loss, together with strength and balance training, may be the most effective approach to improving balance in the obese, potentially reducing the risk of falls and fractures (23). Corbeil et al. reported that the obese moved from a neutral standing position may be at a higher risk for falls than non-obese persons, and that obese individuals are more difficult to destabilize as well as stabilize afterwards (24). Similarly, Ledin and Odkvist and McGraw et al. showed in their studies that adipose tissue accumulation 
in extremely obese teenagers and adults may result in impaired balance and contribute to falls $(25,26)$.

Cruz-Gómez et al. reported a lower quality of life in obese individuals when compared with their normal-weigh counterparts, as well as a higher prevalence of falls and stumbling when walking (16).

Consistently with the above studies, the present study showed improvements in postural activity (the LOS test) and reactions (MCT) and thus the overall postural stability of subjects after weight loss. Other interesting studies on the association between weight and stability include those by Maki et al. and Meyer et al., confirming the strong link between balance and body mass. They claim that the correlation between balance and weight is related to the involvement of plantar mechanoreceptors and skin sensitivity in postural control and the fact that sensitivity of these mechanoreceptors is likely to be inhibited $(27,28)$. The present results support recent research showing that obese adults have higher values of plantar contact area and mean plantar pressure (29). Similarly, Hue et al. stated that obesity was linked to a decrease in balance stability, with the reasons for a strong correlation between postural stability and body weight including, for instance, an association with plantar mechanoreceptors. These are linked to skin sensitivity that influences balance correction. In the obese, the plantar contact area is larger, producing more pressure on the ground and potentially reducing the quality and/or quantity of information for the mechanoreceptors (30).

The above studies clearly demonstrate impaired postural control in obese individuals. The trends in partial results of individual studies are consistent with those in the present study. No comprehensive research covering all parameters and their relationships as contained in the present study is available in the literature. When confronting our results with those available in the literature, it may be concluded that obesity definitely impairs postural skills of an individual and constitutes a risk for trauma to the musculoskeletal system, and that weight loss and waist circumference reduction clearly lead to improved stability, work with the centre of gravity and adaptation to posturally challenging situations in an individual. Thus, both the ability to actively change the centre of gravity depending on particular situation and reactions to a sudden impulse are improved. Their alteration might otherwise lead to falls, injuries and disability.

\section{CONCLUSION}

Even though at the present time obesity and its negative impact on the organism are mainly viewed from a cardiometabolic perspective, it must be realized that obesity also has a considerable negative impact on the musculoskeletal system. Although the consequences are usually less dramatic than cardiac or metabolic complications, the overall quality of life is severely affected. Gradually increasing physical inactivity, weight-bearing joint pain, impaired movement coordination, reduced postural stability and reactions, frequent injuries, need for chronic analgesia use that is not free from adverse effects, and psychological deprivation; these all gradually limit the work, sports and, later, daily living activities of an individual. There is no need to stress that treatment of these conditions is associated with a considerable financial and organizational burden for society as no successful therapy for the negative consequences of obesity on the musculoskeletal system is available as yet. Therefore, these consequences should be minimized by all means possible and, where feasible, prevented by all means possible.

Based on analysis of the study results, it may be concluded that comprehensive group therapy for obesity is clearly beneficial for both weight loss and waist circumference reduction, thus decreasing the overall cardiovascular and metabolic risk, and improving postural parameters, namely postural activity and work with the centre of gravity (velocity, displacement - the LOS test) and reactions (response to a sudden impulse of various intensity - MCT).

\section{Conflict of Interests}

None declared

\section{REFERENCES}

1. Hainer V, et al. Basics of clinical obesitology. 2nd ed. Prague: Grada; 2011. (In Czech.)

2. Owen K. Modern treatment of obesity. Everyday practice guide. Prague: Maxdorf; 2012. (In Czech.)

3. Poděbradská R. Physical activity as a part of overweight and obesity treatment. Rehabil Fyz Lek. 2011;18(2):50-8. (In Czech.)

4. Rothman KJ. BMI-related errors in the measurement of obesity. Int $\mathbf{J}$ Obes [Internet]. 2008 [cited 2014 March 16];32:56-9. Available from: http://www.nature.com/ijo/journal/v32/n3s /full/ijo200887a.html.

5. Wolin KY, Petrelli JM. Obesity. Santa Barbara: Greenwood Publishing Group; 2009.

6. Zeman M, Žák A, Vecka M. News in the treatment and prevention of metabolic syndrome. Postgrad Med. 2007;9(2):124-9. (In Czech.)

7. Dylevský I. Kinesiology: basics of structural kinesiology. Prague: Triton; 2009. (In Czech.)

8. Kolář P, et al. Rehabilitation in clinical practice. Prague: Galén; 2009. (In Czech.)

9. Vařeka I. Postural stability (Part I): terminology and biomechanical principles. Rehabil Fyz Lek. 2002;9(4):115-21. (In Czech.)

10. Kolářová B. Instrumental examination methods for motion evaluation in clinical practice. Olomouc: EZ Centrum; 2012. (In Czech.)

11. NeuroComInternational Inc. Smart EquiTest system operator's manual. version 8. Clackamas: NeuroCom International Inc; 2001.

12. Teasdale N, Hue O, Macotte J, Berrigan F, Simoneau M, Doré J, et al. Reducing weight increases postural stability in obese and morbid obese men. Int J Obes. 2007;31(1):153-60.

13. King AC, Challis JH, Bartok C, Costigan FA, Newell KM. Obesity, mechanical and strength relationships to postural control in adolescence. Gait Posture. 2012;35(2):261-5.

14. NeuroCom ${ }^{\circledR}$ manual. 2012. NeuroCom ${ }^{\circledR}$ [Internet]. [cited 2014 Apr 28]. Available from: http://resourcesonbalance.com/neurocom/products/ SMARTEquiTest.aspx.

15. Ku PX, Abu Osman SA, Yusov A, Wan Abas WA. Biomechanical evaluation of the relationship between postural control and body mass index. J Biomech. 2012;45(9):1638-42.

16. Cruz-Gomez NS, Plascencia G, Villanueva-Padron LA, Jáuregui-Renaud $\mathrm{K}$. Influence of obesity and gender on the postural stability during upright stance. Obes Facts [Internet]. 2011 [cited 2013 May 14];4:212-17. Available from: http://www.karger.com/Article/Pdf/329408.

17. Greve J, Alonso A, Bordini AC, Camanho GL. Correlation between body mass index and postural balance. Clin Sci [Internet]. 2007 [cited 2012 Dec 15];62(6):717-20. Available from: http://www.scielo.br/scielo. php?pid=S1807-59322007000600010\&script=sci_arttext.

18. Handrigan GA, Berrigan F, Hue O, Simoneau M, Corbeil P, Tremblay A, et al. The effects of muscle strength on center of pressure-based measures of postural sway in obese and heavy athletic individuals. Gait Posture. 2012;35(2):88-91.

19. Laughton CA, Slavin M, Katdare K, Nolan L, Bean JF, Kerrigan DC, et al. Aging, muscle activity, and balance control: physiologic changes associated with balance impairment. Gait Posture. 2003;18(2):101-8. 
20. Singh D, Park W, Levy MS, Jung ES. The effects of obesity and standing time on postural sway during prolonged quiet standing. Ergonomics. 2009;52(8):977-86.

21. Colne P, Frelut ML, Peres G, Thoumie P. Postural control in obese adolescents assessed by limits of stability and gait initiation. Gait Posture. 2008;28(1):164-9.

22. Schiepati M, Hugon M, Grasso M, Nardone A, Galante M. The limits of equilibrium in young and elderly normal subjects and in parkinsonians. Electroencephalogr Clin Neurophysiol. 1994;93(4):286-98.

23. Del Porto HC, Pechak CM, Smith DR, Reed-Jones R. Biomechanical effects of obesity on balance. Int J Exerc Sci [Internet]. 2012 [cited 2014 May 14];5(4):301-20. Available from: http://digitalcommons.wku.edu/ ijes/vol5/iss $4 / 1$

24. Corbeil P, Simoneau M, Rancourt D, Tremblay A, Teasdale N. Increased risk for falling associated with obesity: mathematical modeling of postural control. IEEE Trans Neural Syst Rehabil Eng. 2001 Jun;9(2):126-36.

25. Ledin T, Odkvist LM. Effects of increased inertial load in dynamic and randomized perturbed posturography. Acta Otolaryngol. 1993 May;113(3):249-52.

26. McGraw B, McClenaghan BA, Williams HG, Dickerson J, Ward DS. Gait and postural stability in obese and nonobese prepubertal boys. Arch Phys Med Rehabil. 2000;81(4):484-9.
27. Maki BE, Perry SD, Norrie RG, McIlroy WE. Effect of facilitation of sensation from plantar foot-surface boundaries on postural stabilization in young and older adults. J Gerontol A Biol Sci Med Sci. 1999 Jun;54(6):M281-7.

28. Meyer PF, Oddsson LI, De Luca CJ. The role of plantar cutaneous sensation in unperturbed stance. Exp Brain Res. 2004;156(4):505-12.

29. Birtane M, Tuna $H$. The evaluation of plantar pressure distribution in obese and non-obese adults. Clin Biomech [Internet]. 2004 [cited 2014 March 10];19(10):1055-9. Available from:http://www.sciencedirect.com/ science/article/pii/S02680033040 01627.

30. Hue O, Simoneau M, Marcotte J, Berrigan F, Doré J, Marceau P, et al. Body weight is a strong predictor of postural stability. Gait Posture [Internet]. 2007 [cited 2014 Apr 9];26:32-8. Available from: http://ac.els-cdn. com/S0966636206001470/1-s2.0-S0966636206001470-main.pdf? tid= 50bd4474 -c01b-11e3-b86100000aab0f6c\&acdnat=1397071110 b866b73c0defd0e807c839b8d06c 01de.

Received March 21, 2016 Accepted in revised form November 30, 2017 